\title{
CRESCIMENTO VEGETATIVO E CARACTERÍSTICAS DOS FRUTOS DE CINCO CULTIVARES DE ABACAXI NA REGIÃO DE BAURU-SP ${ }^{1}$
}

\author{
ALOÍSIO COSTA SAMPAIO², TEREZINHA DE FÁTIMA FUMIS ${ }^{3}$, SARITA LEONEL ${ }^{4}$
}

RESUMO- Avaliaram-se o crescimento vegetativo e a produção de cultivares novas e tradicionais de abacaxi na região de Bauru. As cultivares estudadas foram: 'Smooth Cayenne', 'Jupi', 'Imperial', 'Gold' e 'Gomo de Mel'. As mudas foram obtidas por cultura de tecidos pela empresa BIONOVA a partir de plantasmatrizes cedidas pela Embrapa Mandioca e Fruticultura Tropical e por produtores comerciais e passaram por um período de 100 dias de aclimatação em telado acondicionadas em tubetes. O plantio no campo foi realizado em canteiros revestidos com 'mulching' plástico preto em janeiro de 2007. O delineamento foi em blocos casualizados, com quatro repetições. Cada parcela apresentou 32 plantas, e o sistema de plantio foi em linhas duplas, no espaçamento $0,30 \times 0,50 \times 1,0 \mathrm{~m}$. A avaliação dos tratamentos foi baseada na determinação do nível de crescimento vegetativo (massa e comprimento da folha ' $\mathrm{D}$ ' e diâmetro do caule) e nas características físicas dos frutos. Os resultados obtidos foram submetidos à análise de variância e ao teste de Tukey, a 5\% de probabilidade. Nas condições ambientais da região de Bauru (SP), e considerando o uso de mudas obtidas por cultura de tecidos, os resultados deste trabalho indicaram a superioridade das cultivares 'Gold', 'Smooth Cayenne' e 'Jupi' em relação a 'Imperial' e 'Gomo de Mel', com crescimento vegetativo maior e produção de frutos mais pesados.

Termos para Indexação: Ananas comosus var. comosus, 'Smooth Cayenne', 'Gold', 'Imperial', 'Jupi' e 'Gomo de Mel'.

\section{VEGETATIVE GROWTH AND FRUIT CHARACTERISTICS OF FIVE CULTIVARS OF PINEAPPLE IN THE BAURU REGION}

\begin{abstract}
It was evaluated the vegetative growth and the productivity of new and traditional cultivars of pineapple in the Bauru region. The cultivars studied were: 'Smooth Cayenne', 'Jupi', 'Imperial', 'Gold' and 'Gomo de Mel'; The saplings were obtained via tissue culture by BIONOVA company from mothers-plants provided by EMBRAPA/CNPMF and commercial producers, been transplanted to cylindrical containers with organic substrate, where they were kept for 100 days. The field planting was developed in seedbeds covered with 'mulching' black plastic in January, 2007, where the experimental design was in randomized blocks with four replicates. Each experimental plot was consisted by 32 plants, and the planting was made in double lines in the spacing of $0,30 \times 0,50 \times 1,0 \mathrm{~m}$. The evaluation of treatments was based on level of vegetative growth determination (mass, length of ' $\mathrm{D}$ ' leaf and stem diameter) and on the fruits physical characteristics. The results were subjected to analysis of variance and Tukey's test at the $5 \%$ level. In the environment conditionsof Bauru region (SP), and considering the use of seedlings obtained via tissue culture, the results of this work indicate the superiority of Gold', 'Smooth Cayenne' and 'Jupi' cultivars in relation to Imperial' and 'Gomo de Mel', with higher vegetative growth and production of the heaviest fruits Index terms: Ananas comosus var. comosus, 'Smooth Cayenne', 'Gold', 'Imperial', 'Jupi' and 'Gomo de Mel'.
\end{abstract}

\footnotetext{
1. Trabalho 190-10). Recebido em: 16-08-2010. Aceito para publicação em: 02-08-2011.

${ }^{2}$ Prof. Adjunto do Dept $^{\circ}$ de Ciências Biológicas, Universidade Estadual Paulista - UNESP,- FC/Bauru-SP e do Curso de Pós-graduação em Horticultura - FCA/Botucatu-SP. E-mail: aloisio@fc.unesp.br

${ }^{3}$ Prof $^{a}$. Assistente/Doutora, Dept ${ }^{\circ}$ de Ciências Biológicas, Universidade Estadual Paulista - UNESP- FC/Bauru-SP. E-mail: tffumis@fc.unesp.br

${ }^{4}$ Prof ${ }^{a}$.Adjunto, Dept ${ }^{\circ}$. de Produção Vegetal, Universidade Estadual Paulista - UNESP-FCA/Botucatu-SP. E-mail: sarinel@fca.unesp.br
} 


\section{INTRODUÇÃO}

O abacaxizeiro (Ananas comosus var. comosus) é, provavelmente, originário da região compreendida entre $15^{\circ} \mathrm{Ne} 30^{\circ} \mathrm{S}$ de latitude e $40^{\circ} \mathrm{L}$ e $60^{\circ} \mathrm{W}$ de longitude, o que inclui as zonas Central e Sul do Brasil, o nordeste da Argentina e o Paraguai. Estudos de distribuição do gênero Ananas indicam que o seu centro de origem é a região da Amazônia, compreendida entre $10^{\circ} \mathrm{N}$ e $10^{\circ} \mathrm{S}$ de latitude e entre $55^{\circ} \mathrm{L}$ e $75^{\circ} \mathrm{W}$ de longitude, por se encontrar nela o maior número de espécies consideradas conhecidas até o momento. Assim, a região Norte do Brasil pode ser considerada um segundo centro de diversificação desse gênero (SOUZA et al., 2000). Dados da FAO (2009) indicam o abacaxi como a terceira fruta tropical mais produzida no mundo, sendo o Brasil o segundo maior produtor mundial, superado apenas pela Tailândia. No Brasil, os principais estados produtores são: Pará, Paraíba, Minas Gerais, Bahia, Amazonas e São Paulo (IBGE, 2006).

$\mathrm{O}$ cultivo do abacaxizeiro nas regiões produtoras brasileiras é realizado em escala comercial, quase que exclusivamente com as cultivares Smooth Cayenne e Pérola, o que torna importante, em termos de sustentabilidade da cadeia de produção desta frutífera, a avaliação de novos materiais genéticos, provenientes de cruzamentos controlados e introduções, assim como de mutações naturais que possam vir a apresentar algum potencial econômico no mercado interno e/ou externo.

Segundo Cabral (2000), as principais características desejadas em uma variedade de abacaxi são: crescimento rápido; folhas curtas, largas e sem espinhos; produção precoce de rebentão localizado na base da planta próxima ao solo; produção de filhotes situados a mais de dois centímetros da base do fruto; fruto de casca de cor amarelo-alaranjada, olhos planos, polpa amarela, firme, mas não fibrosa, teor de açúcar elevado, acidez moderada; coroa média a pequena. Associadas a essas características, procuram-se ainda variedades que proporcionem altos rendimentos e que sejam resistentes e/ou tolerantes às principais pragas e doenças que ocorrem nos locais de plantio. De acordo com o autor, é difícil encontrar uma variedade que reúna todas essas características. Assim, recomendase a escolha de variedades para usos específicos, considerando-se o destino da produção e a adaptação aos locais de plantio.

A região de Bauru (SP) foi pioneira na produção de abacaxi no Estado de São Paulo, na década de 60 , representando, atualmente, o segundo principal polo de cultivo, sendo superado pela região de Guaraçaí (SP). No Estado, a única cultivar plantada e que apresenta uma boa adaptação em relação às condições edafoclimáticas é a 'Smooth Cayenne', mas apresenta tendência de redução de seu consumo no mercado interno, em relação à cv. Pérola (BENGOZI, 2006), e é suscetível à fusariose (Fusarium subglutinas f. sp. ananas). Assim sendo, o estudo de adaptação regional de novas cultivares de abacaxi pode representar perspectivas comerciais importantes para os abacaxicultores do Estado de São Paulo. Nesse sentido, objetivou-se, com este trabalho, avaliar o crescimento vegetativo e as características físicas dos frutos de cinco cultivares de abacaxi na região de Bauru.

\section{MATERIAL E MÉTODOS}

O trabalho foi realizado na Fazenda Santa Irene, localizada no Município de Arealva (SP), em área comercial de produção de abacaxi cv. Smooth Cayenne, distante aproximadamente 20 $\mathrm{km}$ do município de Bauru (SP), com coordenadas de $22^{\circ} 21^{\prime} 28^{\prime \prime}$ de latitude sul e $49^{\circ} 01^{\prime} 37^{\prime \prime}$ de longitude oeste, e altitude de 620 metros. O clima do local é o tipo Cwa (subtropical seco no inverno, segundo a classificação de Köeppen). O solo da área experimental é classificado como Latossolo Vermelho-Amarelo (LVA), apresentando topografia com declives suaves e textura arenosa. Empregou-se o delineamento em blocos casualizados, composto por cinco tratamentos (cultivares) e quatro repetições, sendo que cada parcela foi formada por 32 plantas, totalizando 160 plantas no bloco e 640 plantas na área total. O sistema de plantio foi em linhas duplas, no espaçamento $0,30 \times 0,50 \times 1,0 \mathrm{~m}$, avaliando-se cinco plantas úteis ao acaso nas linhas internas de cada parcela. As cultivares avaliadas foram: 'Smooth Cayenne', 'Jupi', 'Imperial', 'Gomo de Mel'e 'Gold' (MD-2). Os resultados foram submetidos à análise de variância, e as médias dos tratamentos, comparadas pelo teste de Tukey, a 5\% de probabilidade.

As matrizes foram obtidas da Embrapa Mandioca e Fruticultura Tropical e de produtores comerciais. As mudas foram produzidas através da micropropagação in vitro das plantas-matrizes pela Empresa de Biotecnologia BIONOVA de Ribeirão Preto (SP), com a finalidade de aumentar a produção e a uniformidade das mudas. As mudas foram transplantadas para tubetes com substrato orgânico e mantidas no interior de estufa telada, por um período de 100 dias (outubro/06 a janeiro/07). Após este período, foi realizado o plantio em canteiros previamente adubados e revestidos com 'mulching' plástico preto. $\mathrm{O}$ filme plástico teve por 
objetivo evitar um possível assoreamento com terra no centro da roseta ("olho da muda") foliar, em função do período de precipitações intensas. Esta técnica promove efeitos positivos no desenvolvimento vegetativo por propiciar maior retenção de umidade, aumento da temperatura do solo e controle de plantas daninhas (REBOLLEDO-MARTINEZ, 2005).

As práticas culturais, como calagem, adubações, tratamentos fitossanitários e ensacamento dos frutos, foram tradicionalmente empregadas na região de Bauru (SP), levando em consideração os resultados da análise química do solo (Tabela 1).

As medidas de diâmetro do caule foram realizadas com auxílio de um paquímetro digital (março/08), em cinco plantas selecionadas ao acaso nas linhas internas de cada parcela. Para análise foliar, foram coletadas duas folhas ' $\mathrm{D}$ ' por parcela e enviadas ao Departamento de Solos da FCA/ Botucatu-SP. As medidas de peso da biomassa fresca e de comprimento foram realizadas em cinco folhas 'D' nas linhas internas de cada parcela (abril/08).

A indução floral das plantas foi realizada em 9 de maio de 2008, após as 17horas, utilizando-se de ethephon $\left(\right.$ Ethrel $^{\circledR}$ ) na dose de $5 \mathrm{~mL} \mathrm{~L}^{-1}$. Para a avaliação da produtividade, colheram-se cinco frutos ao acaso, nas linhas internas de cada parcela, quando os mesmos se encontravam com a parte basal dos frutos começando a passar da cor verde-escura para a cor verde-clara a amarela (sem atingir mais que duas fileiras de frutilhos), respectivamente (CEAGESP, 2003). As datas de colheitas para cada cultivar foram: 'Jupi' (1'-12-08), 'Gold' (03-12-08), 'Smooth Cayenne' (04-12-08), 'Gomo de Mel' (05-12-08) e 'Imperial' (16-12-08). As coroas foram destacadas manualmente dos frutos e pesadas individualmente e em conjunto com os frutos. Após a pesagem, os frutos foram medidos quanto ao comprimento e ao perímetro (posição mediana do fruto), com auxílio de uma fita métrica.

Durante o período experimental em campo, foram obtidos os dados climáticos de precipitação e temperaturas mínima e máxima mensal nos anos de 2007 e 2008 (Figuras 1 e 2), junto ao Instituto de Pesquisas Meteorológicas (IPMET) da Unesp de Bauru (SP).

\section{RESULTADOS E DISCUSSÃO}

A cultivar Jupi apresentou os maiores pesos e comprimento da folha ' $\mathrm{D}$ ', não diferindo significativamente apenas da 'Gold' quanto ao peso da referida folha (Tabela 2). Os menores valores foram observados na cv. Gomo de Mel, sendo que esta cultivar não diferiu estatisticamente da 'Imperial'.
O peso médio da folha ' $\mathrm{D}$ ', as mais jovens entre as adultas e as mais ativas fisiologicamente entre todas as folhas, segundo vários autores (PY et al., 1984; CHAN; LEE, 2000) são uma variável importante na avaliação do desenvolvimento das plantas e, consequentemente, na definição do momento de se realizar a indução floral. De acordo com Py et al. (1984), para obtenção de frutos de 'Smooth Cayenne' com 1,5 kg, deve-se realizar a indução floral em plantas com $80 \mathrm{~cm}$ de comprimento e 70 gramas de peso da matéria fresca da folha ' $D$ '.

O diâmetro do caule é uma variável observada por alguns produtores para definir a época adequada para a indução floral artificial. As plantas das cultivares Gold, Smooth Cayenne e Jupi apresentaram os maiores valores médios, sendo que as duas últimas não diferiram das demais cultivares estudadas.

De acordo com Souza (1999), a análise química dos tecidos foliares é o instrumento que permite uma avaliação mais eficiente do estado nutricional da planta, desde que existam informações bem definidas sobre órgãos a serem amostrados e épocas de amostragem, associados a padrões de referência adequados, para a interpretação dos resultados analíticos de cada nutriente. As concentrações adequadas de nutrientes na folha 'D', presentes na literatura, não distinguem as cultivares (Tabela 3), fator que deve ser considerado, principalmente nas nacionais.

A análise foliar realizada neste estudo indicou diferenças na concentração de macro e micronutrientes presentes nas folhas das diferentes cultivares (Tabela 3), o que pode sugerir a necessidade do desenvolvimento de uma calibração específica da exigência nutricional para cada cultivar de abacaxi. Como os teores de potássio se encontravam abaixo do recomendado por Lacoeuilhe (1984), citado por Souza (1999), para todas as cultivares em estudo, realizou-se adubação de cobertura com 10 gramas de cloreto de potássio por planta, na $1^{\text {a }}$ quinzena de abril $/ 08$.

Teixeira et al. (2007) elaboraram algumas normas preliminares de sistema integrado de diagnóstico e recomendação (DRIS) e níveis críticos de nutrientes nas folhas (NCL), a partir de um banco de dados de quatro experimentos de fertilização de abacaxi 'Smooth Cayenne' realizados na região de Bauru (SP). Os NCLs para N, P, K, Ca e Mg foram 12,$0 ; 0,92 ; 21,4 ; 4,0$ e $2,8 \mathrm{~g} \mathrm{Kg}^{-1}$, respectivamente, sendo similares àqueles presentes na literatura, à exceção de $\mathrm{Ca}$ e $\mathrm{Mg}$, cujos valores são mais altos para condições locais. Guarçoni et al. (2007) esti- 
maram os teores foliares de N, P e K em 1,54; 0,20 e 3,72\%, respectivamente, para a produção máxima do abacaxi 'Gold' (68,6 t ha' $\left.\mathrm{t}^{-1}\right)$.

O mercado interno de abacaxi no Brasil dá preferência para frutos graúdos e, nesse sentido, os aspectos que interferem no crescimento vegetativo das plantas no campo são determinantes para o sucesso econômico da atividade, pois há uma correlação positiva entre o tamanho da planta (peso de folha 'D') e o peso dos frutos (PY et al., 1984). Desse modo, a produtividade determinada através do peso médio dos frutos está diretamente relacionada às variáveis: manejo nutricional, época de plantio, cultivar, uso de irrigação, tipo de muda, etc.

As cultivares Gold e Jupi apresentaram frutos de maior massa e tamanho (perímetro e comprimento), diferindo significativamente das cultivares Smooth Cayenne, Imperial e Gomo de Mel (Tabela 4). Como o mercado interno de fruta fresca dá preferência por frutos médios a grandes (peso $>1,5 \mathrm{~kg}$ ), mesmo as cultivares de melhor desempenho neste trabalho não atingiram este valor desejado. Como mudas de abacaxi produzidas in vitro normalmente apresentam crescimento vegetativo e produção inferiores aos obtidos com mudas convencionais, os resultados poderão ser significativamente superiores no ciclo seguinte conduzido com mudas convencionais dos tipos filhote e rebentões.

Cunha et al. (2007), ao avaliarem genótipos de abacaxi resistentes à fusariose, concluíram que a 'Imperial' e o cruzamento 'PE x SC60' apresentaram os menores valores de massa do fruto, ou seja, 0,68 e $0,67 \mathrm{~kg}$, respectivamente. Já as cultivares Jupi e Gold destacaram-se em relação ao tamanho, com valores de 1,21 e $1,38 \mathrm{~kg}$, respectivamente. A cultivar Imperial apresentou peso do fruto de $0,67 \mathrm{~kg}$ (Tabela 4), bem inferior ao mencionado pelos técnicos da Embrapa, que descreveram o material com potencial de produzir frutos com coroa de $1,67 \mathrm{~kg}$, usando mudas convencionais e nas condições dos tabuleiros costeiros da Bahia (Embrapa, 2003).

De acordo com Usberti Filho et al. (1999), a cultivar Gomo de Mel possui tamanho pequeno, facilitando o manuseio, a embalagem e o transporte, sendo ainda adequada para consumo individual, pois apresenta frutilhos ('olhos') soldados menos fortemente entre si, podendo ser destacáveis do fruto, o que proporciona maior facilidade no consumo, não necessitando ser descascada. Em função do pequeno tamanho, baixa oferta de frutos, ser comercializada como fruta exótica e apresentar o mesmo custo de produção das outras cultivares presentes no mercado, os seus frutos possuem valores de venda ao consumidor final bem superiores.

As cultivares Gomo de Mel e Imperial produziram um número médio de filhotes na base dos frutos, respectivamente, de quatro e nove mudas, que provavelmente induziram uma redução no tamanho dos frutos. Este efeito pode ter sido influenciado pelo sistema de cultura de tecidos, que produz mudas mais sadias e homogêneas, mas também com menor quantidade de reservas e, muitas vezes, ficam sob efeito colateral de fitorreguladores empregados para sua multiplicação in vitro.

TABELA 1 - Análise química do solo da área experimental, Arealva-SP.

\begin{tabular}{|c|c|c|c|c|c|c|c|c|}
\hline $\begin{array}{c}\mathrm{pH} \\
\left(\mathrm{CaCl}_{2}\right)\end{array}$ & $\begin{array}{l}\mathrm{MO} \\
\mathrm{g} / \mathrm{dm}^{3}\end{array}$ & $\begin{array}{c}\mathrm{P}(\text { resina }) \\
\mathrm{mg} / \mathrm{dm}^{3}\end{array}$ & $\mathrm{~K}$ & $\begin{array}{c}\mathrm{Ca} \\
\mathrm{mr}\end{array}$ & $\mathrm{Mg}$ & $(\mathrm{H}+\mathrm{Al})$ & $\mathrm{T}(\mathrm{CTC})$ & $\begin{array}{l}\mathrm{V} \\
(\%)\end{array}$ \\
\hline 5,3 & 13 & 3 & 0,6 & 11 & 8 & 15 & 35 & 56 \\
\hline
\end{tabular}

TABELA 2 - Comparação do crescimento vegetativo de plantas de cinco cultivares de abacaxi aos 13/14 meses após o plantio, Arealva-SP.

\begin{tabular}{lccrc}
\hline Cultivares & Massa de folha ' $\mathrm{D}$ ' & Comprimento de folha 'D' & Diâmetro do caule \\
\hline \multicolumn{1}{c}{$(\mathrm{g})$} & $(\mathrm{cm})$ & $(\mathrm{mm})$ \\
\hline Gold & $56,75 \mathrm{ab}$ & $75,75 \mathrm{~b}$ & $77,25 \mathrm{a}$ \\
Jupi & $66,25 \mathrm{a}$ & $93,75 \mathrm{a}$ & $67,25 \mathrm{ab}$ \\
S. Cayenne & $43,50 \mathrm{bc}$ & $70,25 \mathrm{bc}$ & $74,00 \mathrm{ab}$ \\
Imperial & $34,00 \quad \mathrm{~cd}$ & 63,25 & $\mathrm{~cd}$ & $65,25 \mathrm{~b}$ \\
Gomo de Mel & $25,50 \quad \mathrm{~d}$ & $58,75 \quad \mathrm{~d}$ & $65,50 \mathrm{~b}$ \\
\hline CV $(\%)$ & 7,37 & 14,03 & 7,26 \\
\hline
\end{tabular}

Valores com a mesma letra na coluna não diferem entre si, pelo teste de Tukey, a $5 \%$ de probabilidade. 
TABELA 3 - Teor de macro e micronutrientes de folhas ' $\mathrm{D}$ ' (\%) coletadas aos catorze meses após o plantio de cinco cultivares de abacaxi, Arealva-SP.

\begin{tabular}{lcccccccccc}
\hline Cultivares & $\mathrm{N}$ & $\mathrm{P}$ & $\mathrm{K}$ & $\mathrm{Ca}$ & $\mathrm{Mg}$ & $\mathrm{B}$ & $\mathrm{Cu}$ & $\mathrm{Fe}$ & $\mathrm{Mn}$ & $\mathrm{Zn}$ \\
\hline Gold & 1,0 & 0,10 & 1,7 & 0,2 & 0,18 & 11 & 4 & 51 & 121 & 21 \\
Jupi & 1,3 & 0,13 & 1,8 & 0,3 & 0,21 & 29 & 6 & 47 & 216 & 22 \\
S. Cayenne & 1,0 & 0,12 & 1,7 & 0,2 & 0,19 & 12 & 5 & 79 & 170 & 16 \\
Imperial & 1,3 & 0,12 & 1,6 & 0,2 & 0,18 & 14 & 5 & 41 & 132 & 20 \\
Gomo de Mel & 1,2 & 0,13 & 2,0 & 0,2 & 0,21 & 11 & 4 & 54 & 148 & 21 \\
\hline \hline
\end{tabular}

$\begin{array}{lllllll}\text { IRFA }^{1} & >1,2 & >0,08 & >2,8 & >0,1 & >0,1 & -\end{array}$

${ }^{1}$ Concentrações ideais no momento da indução floral. Fonte: Souza, 1999.

TABELA 4 - Massa fresca e tamanho dos frutos de cinco cultivares de abacaxi, Arealva-SP.

\begin{tabular}{|c|c|c|c|c|c|c|c|}
\hline Cultivares & \multicolumn{2}{|c|}{ Massa do fruto $(\mathrm{kg})$} & & \multicolumn{2}{|c|}{$\begin{array}{l}\text { Perímetro } \\
\text { (cm) }\end{array}$} & $\begin{array}{c}\text { Comprimento } \\
(\mathrm{cm})\end{array}$ & $\begin{array}{c}\text { Massa fresca } \\
\text { coroa }(\mathrm{g})\end{array}$ \\
\hline Gold & $1,47 \mathrm{a}$ & $1,30 \mathrm{a}$ & & $37,24 \mathrm{a}$ & & $15,38 \mathrm{a}$ & $165,0 \mathrm{a}$ \\
\hline Jupi & $1,40 \mathrm{a}$ & $1,26 \mathrm{a}$ & & $36,49 \mathrm{a}$ & & $17,18 \mathrm{a}$ & $137,8 \mathrm{a} \mathrm{b}$ \\
\hline S. Cayenne & $1,34 \mathrm{a}$ & $1,22 \mathrm{a}$ & & 27,11 & $\mathrm{~b}$ & $15,38 \mathrm{a}$ & $121,3 \quad b$ \\
\hline Imperial & $0,67 \mathrm{~b}$ & 0,60 & b & 31,38 & $\mathrm{~b}$ & $12,10 \quad b$ & 68,8 \\
\hline Gomo de Mel & $0,56 \mathrm{~b}$ & 0,45 & $\mathrm{~b}$ & 29,27 & $\mathrm{~b}$ & $8,50 \quad \mathrm{~b}$ & $108,8 \quad \mathrm{~b}$ \\
\hline$\overline{\mathrm{CV}(\%)}$ & & & & 3,75 & & 9,04 & 14,47 \\
\hline
\end{tabular}

Valores com a mesma letra na coluna não diferem entre si, pelo teste de Tukey, a 5\% de probabilidade.

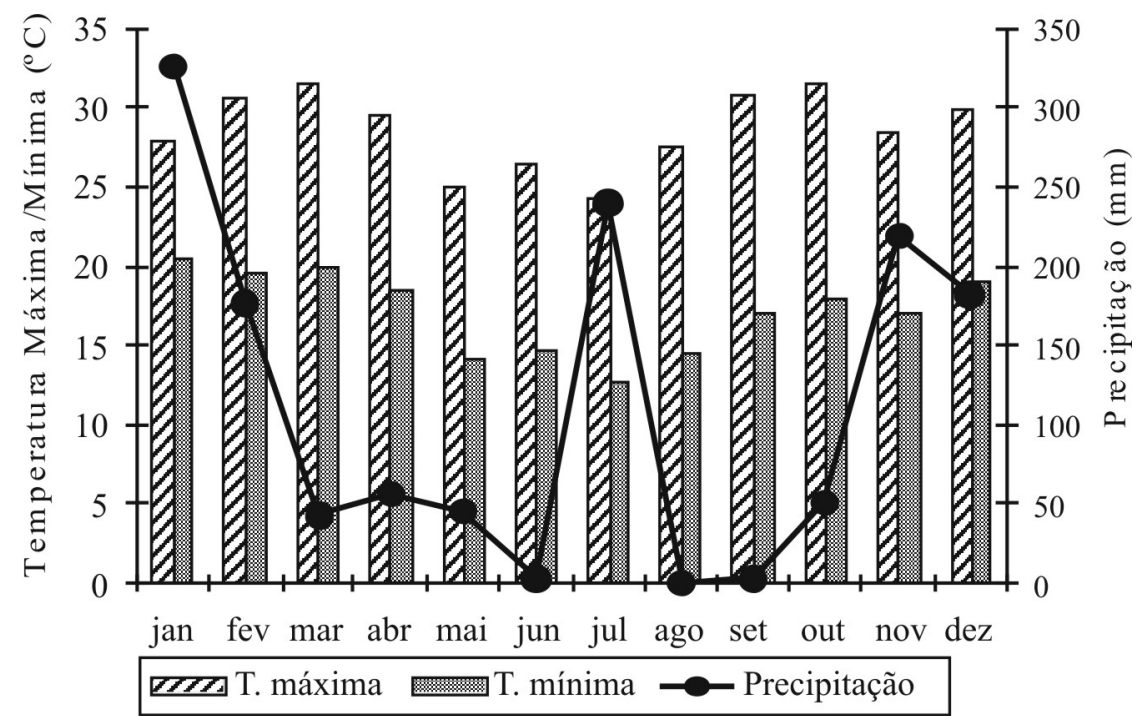

Fonte: IPMET/UNESP/BAURU-SP, 2007.

FIGURA 1- Precipitação e temperaturas médias mínima e máxima mensal, durante o ano de 2007. 


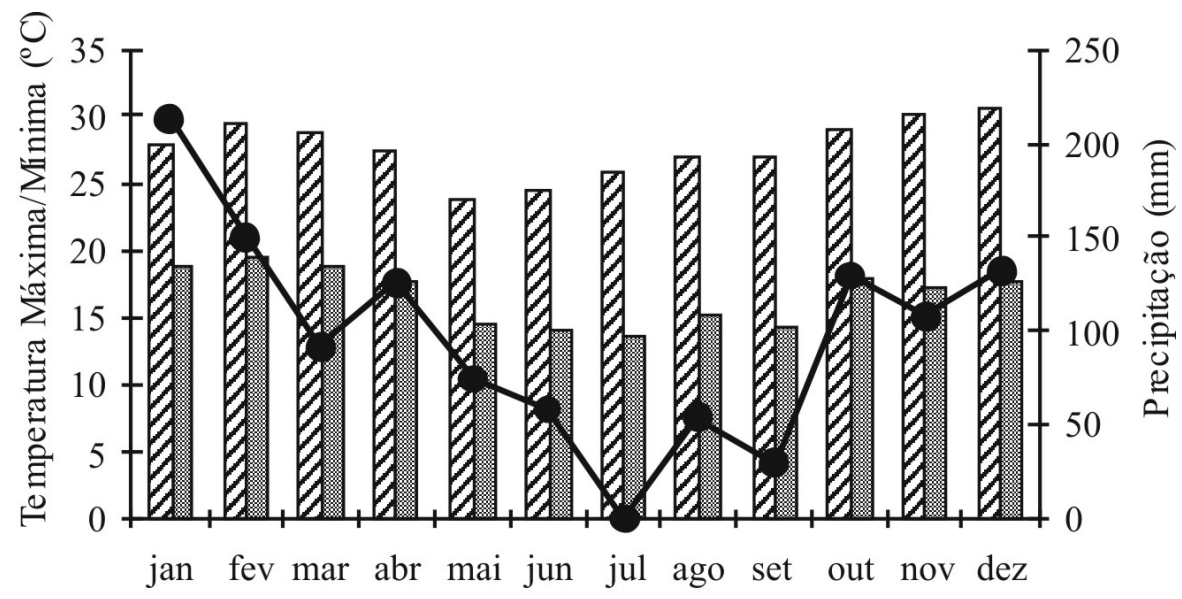

$\square$ T. máxima

Fonte: IPMET/UNESP/BAURU-SP, 2008.

FIGURA 2 - Precipitação e temperaturas médias mínima e máxima mensal, durante o ano de 2008.

\section{CONCLUSÃO}

Nas condições ambientais da região de Bauru-SP, e considerando o uso de mudas obtidas por cultura de tecidos, os resultados deste trabalho indicam: superioridade das cultivares Gold, Smooth Cayenne e Jupi em relação à Imperial e Gomo de Mel, com crescimento vegetativo maior e produção de frutos mais pesados; necessidade de se determinar uma calibração nutricional específica para as cultivares comerciais de abacaxi.

\section{REFERÊNCIAS}

BENGOZI, F. J. Procedência, sazonalidade e qualidade físico-química do abacaxizeiro comercializado na Ceagesp - São Paulo. 2006. $134 \mathrm{f}$. Dissertação (Mestrado em Horticultura) - Faculdade de Ciências Agronômicas, Universidade Estadual Paulista, Botucatu, 2006.

CABRAL, J. R. S. Variedades. In: REINHARDT, D. H. et al. (Org.) Abacaxi produção: aspectos técnicos. Brasília: Embrapa, 2000, p. 15-18.

CEAGESP - Centro de Qualidade em Horticultura. Programa brasileiro para modernização da horticultura: normas de classificação do abacaxi: São Paulo, 2003. (Documentos, 24).
CHAN, H. E.; LEE, H. K. Breeding for early fruiting in pineapple. Acta Horticulturae, The Hague, v. 291 , p. $60-70,2000$.

CUNHA, G. A. P. et al. Avaliação de genótipos de abacaxi resistentes a fusariose em Coração de Maria, Bahia. Magistra, Cruz das Almas, v. 19, n. 3, p. 219-223, 2007.

EMBRAPA. Centro Nacional de Pesquisa de Mandioca e Fruticultura. Disponível em: $<$ http://www. cnpmf.embrapa.br/extra_2003/015_Imperial_\%20 PB_14_05.htm>. Acesso em: 18 maio 2009.

FAO. FAOSTAT Database. Roma, 2009. Disponível em: <http://faostat.fao.org/faostat/ form?collection=Production.Crops.Primary\&Dom ain $=$ Production \&servlet $=1 \&$ hasbulk $=0$ \&language $=\mathrm{EM}>$. Acesso em: 20 abr. 2009.

GUARÇONI, A.M. et al. Leaf nutrient concentration and yield of 'MD2' pineapple as a function of NPK fertilization. In: SIMPOSIO INTERNACIONAL DE ABACAXI, 6., 2007, João Pessoa. Anais...

IBGE. Sistema IBGE de recuperação automática SIDRA, 2006. Disponível em: <http//:www.sidra. ibge.gov.br.>. Acesso em: 20 abr. 2009. 
LACOEUILHE, J.J. Ananás. In: MARTIN-PRÉVEL, P.; GAGNARD, J.; GAUTIER, P. L'analyse végétale dans le contrôle de l'alimentation des plantes. Paris: Lavoisier, 1984. p. 675-694. (Technique et Documentation)

PY, C.; LACOEUILHE, J. J.; TEISON, C. L'ananas, as culture, sés produits. Paris: G.P. Maisonneuve et Larose et A.C.C.T., 1984.

REBOLLEDO-MARTINEZ, A. Growth analysis for three pineapple cultivars grown on plastic mulch and bare soil. Interciência, Caracas, v.30, n.12, p. 758-763, 2005

SOUZA, L. F. S. Exigências edáficas e nutricionais. In: CUNHA, G.A.P.C.; CABRAL, J. R. S. C.; SOUZA, L. F. S. (Org.). O abacaxizeiro: cultivo, agroindústria e economia. Brasília: Embrapa, 1999. p. 67-82.
SOUZA, L. F. S.; REINHARDT, D. H.; CABRAL, J. R. S. Introdução. In: REINHARDT, D. H.; SOUZA, L. F. S.; CABRAL, J. R. S. (Org.). Abacaxi produção: aspectos técnicos. Brasília: Embrapa, 2000. p. 15-18.

TEIXEIRA, L. A. J.; QUAGGIO, J. A.; ZAMBROSI, F. C. B. Normas preliminares de DRIS para abacaxi 'Smooth Cayenne' e derivação de níveis críticos de concentrações foliares de nutrientes. IN: SIMPÓSIO INTERNACIONAL DE ABACAXI, 6., 2007. Anais...

USBERTI FILHO, J. A. et al. Abacaxi gomo-demel. Campinas: Instituto Agronômico, 1999. 5 p. Disponível em: <http://200.136.175.13/homeiac/ produtos/abacaxi.htm>. Acesso em: 14 jul. 2009. 